\title{
Astrochemistry revealed
}

\section{Stephen Lepp}

The Chemically Controlled Cosmos: Astronomical Molecules from the Big Bang to Exploding Stars. By T. W. Hartquist and D. A. Williams. Cambridge University Press: 1995. Pp. 169. £19.95, $\$ 39.95$.

AsTRONOMERS study regions of the Universe that cannot be visited, inferring what they know about them from the light and particles reaching Earth. The most precise information comes in the form of light emitted by molecules at particular frequencies with an intensity dependent on the local environment, such as the density, temperature or radiation field. This makes such molecules excellent indicators of the physical conditions in astrophysical environments.

How molecules form and emit radiation is a subject that has long been neglected in the popular literature on astronomy. Hartquist and Williams have rectified the situation. As the title of their new book suggests, they not only explain how molecules may be used to probe the physical conditions, but they also show how molecules control the environment and evolution of astrophysical objects.

The presentation may at times be a bit difficult for a truly non-technical reader, although it should be accessible to anyone who regularly reads $S c i$ entific American or the News and Views section of Nature. It would make an excellent introduction to molecular astrophysics for a beginning graduate student, particularly if supplemented by the selected references at the end of each chapter. Astronomers, chemists or any scientist curious about the chemistry of astrophysical objects will find it a useful resource. I suspect most readers will be surprised at just how pervasive molecules are in the Universe, so much so that the book is nearly an overview of astronomy, covering such wideranging topics as the

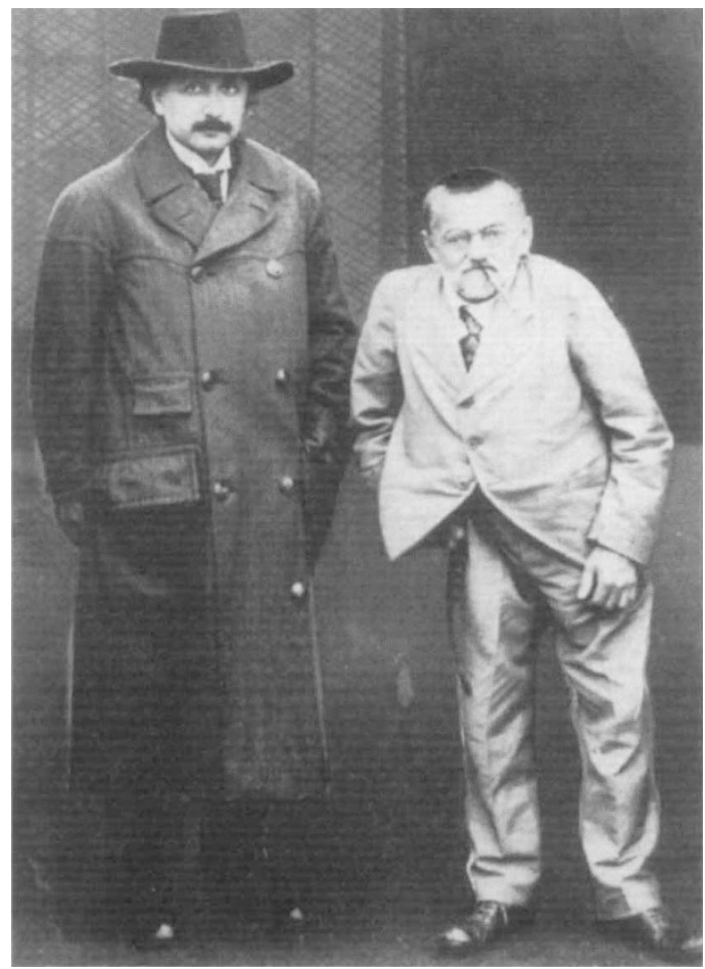

ALBERT Einstein with the American electrical engineer C. P. Steinmetz, c.1920. The picture is taken from Essential Einstein edited by A. B. Eddington. This slender glossy paperback contains 60 duotone photographs of Einstein, some famous, others less well known. Accompanied by quotations from his letters, speeches and recorded conversations, they provide a revealing portrait of the scientific, political and humane sides of the great man. Pomegranate, $£ 22.95$, £15.95.

\section{Wide illumination}

\section{Frank McCapra}

Photobiology. By E. Kohen, R. Santus and J. G. Hirschberg. Academic: 1995. Pp. 506. £54, \$69.95.

Photobiology in its entirety is rarely given its proper place in university courses. The range of topics is great, and their study often leads to mutually exclusive specialization, despite the self-evident unifying presence of the action of light. As the authors of this book point out in their preface, photobiology is a truly multidisciplinary subject that touches on every aspect of life. A wide-ranging overview is therefore particularly valuable.

All aspects of the action of light on biological systems are covered here, but the treatment is uneven. Although the early chapters on the nature of light and some basic photophysics make for a convenient single-volume reference, similar and more detailed treatments of these topics can be found elsewhere. In any case, later cross-referencing to this background material is rarely provided, so these early chapters are a missed opportunity. What is more, details on the natural successor to such information - the photochemistry of relevant organic molecules - are almost wholly lacking. One might have expected, for example, a discussion in the chapter on vision of current thinking on cis-trans isomerism and models for the molecular basis of colour vision. (It seems that the authors were each responsible for isolated parts of the book and were self-effacing in referring to their own research.)

The chapters on vision and bioluminescence are disappointing: they fail to include the most up-to-date work and give insufficient details of the underlying organic chemistry. There is an effective emphasis on medical aspects, reflected by the large bibliographies for these chapters (in this respect some others in the book are seriously deficient), with many unpleasant, and not entirely necessary, photographs of diseases caused by or treated by light.

Nevertheless, the authors clearly bring out the importance of the interaction of light with the biosphere. They give a good picture of the enormous range of activity in this field and a fairly up-to-date description of discoveries and prospects in such research topics as environmental photobiology, photosynthesis, photocarcinogenesis and phototherapy. In short, it amounts to a reasonably successful, compact compendium.

Frank McCapra is at the School of Chemistry, University of Sussex, Falmer, Brighton BN1 9QJ, UK. 